\title{
A numerical scheme for Fredholm integral equations
}

\author{
A.R. Nazemi ${ }^{1, *}$, M.H. Farahi ${ }^{2}$ \\ 1 Department of Applied Mathematics, School of Mathematical Sciences, \\ Shahrood University of Technology, Shahrood, Iran. \\ 2 Department of Mathematical Sciences, Ferdowsi University of Mashhad, \\ Iran.
}

\begin{abstract}
A different numerical method for nonlinear Fredholm integral equations of the second kind with the continuous kernel is considered. The main idea is to convert the integral equation problem into an optimization problem. Then by using an embedding method, the class of admissible trajectories is replaced by a class of positive Borel measures. The optimization problem in measure space is then approximated by a finite dimensional linear programming (LP) problem. Some examples demonstrate the effectiveness of the method.
\end{abstract}

Keywords: Fredholm integral equation; Functional space; Measure space; Approximation; Linear programming.

Mathematics Subject Classification 2010: 45D05, 90C05, 28Axx.

\section{Introduction}

In this paper, we study the following nonlinear Fredholm integral equation of the second kind

$$
u(x)=f(x)+\int_{a}^{b} k(t, x, u(t)) d t, \quad x \in[a, b],
$$

where $u(x)$ is an unknown function, $f(x)$ and $k(t, x, u(t))$ are given continuous functions defined, respectively on $[a, b]$ with $k(t, x, u)$ nonlinear in $u$. Many problems in engineering and basic sciences can be transformed into Fredholm integral equations of the second kind $[1,3,7,8,14,19,28]$. There are many works on developing and analyzing numerical

\footnotetext{
${ }^{*}$ Correspondence to: A.R. Nazemi, Department of Applied Mathematics, School of Mathematical Sciences, Shahrood University of Technology, P.O. Box 3619995161-316, Tel/Fax No :0098-273-3392012, Shahrood, Iran. Email: nazemi20042003@yahoo.com

${ }^{\dagger}$ Received: 2 October 2010, revised: 21 November 2010, accepted: 1 February 2011.
} 
methods for solving Fredholm integral equations $[2,4-6,12,13,15-17,20]$. We assume throughout this paper that the integral equation (1) has a unique solution. Conditions for existence and uniqueness of the solution for the problem (1.1) is described in [14].

Motivated by the above discussions, in this paper, we present the optimization technique for solving problem (1) based on the measure theory method [25]. The advantages of the proposed method are in the fact that the method is not iterative, it is self-starting and it is not restricted to differentiable cost functions. Because of these features, this method has been extended to solve a variety of optimal control and optimization problems $[9-11,18,21-23]$.

\section{Moment problem}

Let $\Delta=\left\{x_{0}, x_{1}, \ldots, x_{M}\right\}$ be an equidistance partition of $I=[a, b]$, where $h=x_{i+1}-$ $x_{i}, i=0,1, \cdots, M-1$ is the discretization parameter of the partition. Now, for the partition $\Delta=\left\{x_{0}, x_{1}, \ldots, x_{M}\right\}$ on $I$, the integral equation (1.1) can be discretized in the following form:

$$
\left\{\begin{array}{l}
\int_{a}^{b} k\left(t, x_{0}, u(t)\right) d t-u\left(x_{0}\right)=-f\left(x_{0}\right), \\
\int_{a}^{b} k\left(t, x_{1}, u(t)\right) d t-u\left(x_{1}\right)=-f\left(x_{1}\right), \\
\vdots \\
\int_{a}^{b} k\left(t, x_{M}, u(t)\right) d t-u\left(x_{M}\right)=-f\left(x_{M}\right) .
\end{array}\right.
$$

We define an approximating optimization problem corresponding to the integral equation (1) as follows:

$$
\text { minimize } \quad \int_{a}^{b} g(t, u(t)) d t
$$

subject to

$$
\int_{a}^{b} k\left(t, x_{i}, u(t)\right) d t-u\left(x_{i}\right)=-f\left(x_{i}\right),(i=0,1, \ldots, M),
$$

where $g(t, u(t))$ is a continuously differentiable function and is given.

Proposition 2.1. Finding a solution for the approximating system (2.1) of the integral equation (1.1) is equivalent to find a solution of the optimization problem (2.2)-(2.3).

Proof. The proof is clear, since problem (1.1) has a unique solution.

Definition 2.1. The trajectory function $u(\cdot):[a, b] \rightarrow \mathbb{R}$ is called admissible if it is absolutely continuous and the constrains (2.3) are satisfied. We denote the set of all admissible trajectories by $U_{a d}$ which is also nonempty.

Now integral equation problem (1) is reduced to finding a solution $u \in U_{a d}$ satisfying:

$$
\text { minimize } \quad \int_{a}^{b} g(t, u(t)) d t
$$


subject to

$$
\int_{a}^{b} k_{i} d t=a_{i}, \quad(i=0,1, \cdots, M)
$$

where for simplicity, we denote

$$
a_{i}=u\left(x_{i}\right)-f\left(x_{i}\right) \text {, and } k_{i}=k\left(t, x_{i}, u(t)\right),(i=0,1, \cdots, M) .
$$

In the next section, we proceed to enlarge the set $U_{a d}$.

\section{Metamorphosis}

In general, it may be difficult to characterize the optimal trajectory in $U_{a d}$; necessary conditions are not always helpful because the information that they give may be impossible to interpret. It appears that these situations may become more favorable if the set $U_{a d}$ could somehow be made larger. In the following we use a transformation to enlarge the set $U_{a d}$.

Let $\Omega=I \times U$, where $U$ is a known compact sets in $\mathbb{R}$ such that the trajectory $u$ gets its values for each $x \in I$ in this set, and $C(\Omega)$ is the space of all real-valued continuously differentiable functions on $\Omega$. For each admissible trajectory $u \in U_{a d}$, we correspond the following linear continuous functional

$$
\Lambda: h \longrightarrow \int_{a}^{b} h(t, u(t)) d t, \quad \forall h \in C(\Omega) .
$$

Some aspects of this mapping are useful; it is well defined, and positive.

Proposition 3.1. Transformation $u \rightarrow \Lambda$ of an admissible trajectory in $U_{a d}$ into the linear mapping $\Lambda$ defined in (3.1) is an injection.

Proof. We must show that if $u_{r} \neq u_{q}$ then $\Lambda_{r} \neq \Lambda_{q}$. Indeed, if $u_{r}$ and $u_{q}$ are different admissible trajectories, then there is a subinterval of $I$, say $N_{L}$, where $u_{r}(t) \neq u_{q}(t)$ for $t \in N_{L}$. A continuous positive function $h$ can be constructed on $I$ so that the right-hand side of equation (3.1) corresponding to $u_{r}$ and $u_{q}$ are not equal. For instance, assume for all $t \in N_{L}$, the function $h$ is positive on the appropriate portion of the graph of $u_{r}(\cdot)$, and zero on $u_{q}(\cdot)$. Then the corresponding linear functionals are not equal.

Thus, solving (2.4)-(2.5) is equivalent to find $\Lambda$ in functional space $C^{*}(\Omega),\left(C^{*}\right.$ is the dual space), such that

$$
\text { minimize } \Lambda(g) \text {, }
$$

subject to

$$
\Lambda\left(k_{i}\right)=a_{i},(i=0,1, \cdots, M) .
$$


By Riesz representation theorem [26], there exists a unique positive Radon measure $\mu$ on $\Omega$ such that

$$
\Lambda(h)=\int_{\Omega} h d \mu=\mu(h), h \in C(\Omega) .
$$

These measures $\mu$ are required to have certain properties which are abstracted from the definition of admissible trajectories. First, from (3.4)

$$
|\mu(h)| \leq T \sup _{\Omega}|h(t, u(t))|,
$$

where $T=b-a$. Hence

$$
\mu(1) \leq T
$$

From (3.3) and (3.4), we see that the measures $\mu$ satisfy

$$
\mu\left(k_{i}\right)=a_{i}, \quad(i=0,1, \cdots, M) .
$$

Next, suppose that $\theta \in C(\Omega)$ does not depend on $u$, that is

$$
\theta\left(t, u_{1}\right)=\theta\left(t, u_{2}\right)
$$

for all $t \in[a, b]$ and $u_{1}, u_{2} \in U$, where $u_{1}(\cdot) \neq u_{2}(\cdot)$. Then the measures $\mu$ must satisfy

$$
\int_{\Omega} \theta d \mu=\int_{a}^{b} \theta(t, u(t)) d t=\alpha_{\theta},
$$

where $u$ is an arbitrary number in the set $U$, and $\alpha_{\theta}$ is the Lebesgue integral of $\theta(\cdot, u)$ over $I$.

Let $M^{+}(\Omega)$ be the set of all positive Radon measures on $\Omega$. We topologize the space $M^{+}(\Omega)$ by the weak*-topology and define the set $Q$ as a subset of $M^{+}(\Omega)$ as follows

$$
Q=S_{1} \cap S_{2} \cap S_{3}
$$

where

$$
\begin{aligned}
& S_{1}=\left\{\mu \in M^{+}(\Omega): \mu(1) \leq T\right\} \\
& S_{2}=\left\{\mu \in M^{+}(\Omega): \mu\left(k_{i}\right)=a_{i},(i=0,1, \cdots, M)\right\} \\
& S_{3}=\left\{\mu \in M^{+}(\Omega): \mu(\theta)=\alpha_{\theta}, \theta \in C(\Omega) \text { independent of } u\right\} .
\end{aligned}
$$

So one may change the problem (3.2)-(3.3) in functional space to the following optimization problem in measure space

$$
\begin{aligned}
& \operatorname{minimize} I(\mu)=\int_{\Omega} d \mu \equiv \mu(g) \\
& \text { subject to }
\end{aligned}
$$

$$
\mu \in Q
$$


Theorem 3.1. The set $Q$ is compact in $M^{+}(\Omega)$.

Proof. The set $S_{1}$ is compact and the set $S_{2}$ can be written as

$$
S_{2}=\bigcap_{i=1}^{M}\left\{\mu \in \mathcal{M}^{+}(\Omega): \mu\left(k_{i}\right)=a_{i}\right\}=\bigcap_{i=1}^{M} W_{i},
$$

where each $W_{i}=\left\{\mu \in \mathcal{M}^{+}(\Omega): \mu\left(k_{i}\right)=a_{i}\right\}$ is closed, because it is the inverse image of a closed set on the real line, the set $\left\{a_{i}\right\}$, under a continuous map. By a similar argument, it is easy to show that $S_{3}$ is closed. Thus $Q$ is a closed subset of the compact set $S_{1}$, and then $Q$ is compact.

Theorem 3.2. The measure-theoretical problem, which consists of finding the minimum of the functional (3.5) over the set $Q$ of $M^{+}(\Omega)$, possesses a minimizing solution $\mu^{*}$, say, in $Q$.

Proof. The proof is clear, since $\mu$ is a linear functional on a compact set $Q$, therefor it attains its minimum.

In the next sections, we shall establish a method for estimating numerically trajectories which approximate the action of the optimal measures.

\section{Approximation to the optimal measure}

In this section, we obtain an approximation to the optimal measure $\mu^{*}$ satisfying in (3.5)-(3.6).

It is clear that the measure theoretical problem (3.5)-(3.6), can be written in the following form

$$
\operatorname{minimize} \quad I(\mu)=\mu(g)
$$

subject to:

$$
\left\{\begin{array}{l}
\mu(1) \leq T \\
\mu\left(k_{i}\right)=a_{i}, \quad(i=0,1, \cdots, M), \\
\left.\mu(\theta)=\alpha_{\theta}, \theta \in C(\Omega) \text { independent of } u\right\}
\end{array}\right.
$$

The minimizing problem of (4.1)-(4.2) is an infinite-dimensional LP problem and we are mainly interested in approximating it. It is possible to approximate the nearly trajectory function of the problem (4.1)-(4.2) by the solution of a finite dimensional LP of sufficiently large dimension.

First we consider the minimization of (4.1) not only over the set $Q$, but also over a subset of it defined by requiring that only a finite number of constraints (4.2) be satisfied. This will be achieved by choosing countable sets of functions whose linear combinations are dense in the appropriate spaces, and then selecting a finite number of them. 
Proposition 4.1. Let $Q(M, G)$ be a subset of $M^{+}(\Omega)$ consisting of all measures which satisfy

$$
\left\{\begin{array}{l}
\mu(1) \leq T, \\
\mu\left(k_{i}\right)=a_{i}, \quad(i=0,1, \cdots, M), \\
\mu\left(\theta_{v}\right)=\alpha_{\theta_{v}}, \quad(v=1,2, \ldots, G) .
\end{array}\right.
$$

As $M$ and $G$ tend to infinity, $\varrho(M, G)=\inf _{Q(M, G)} \mu(g)$ tends to $\varrho=\inf _{Q} \mu(g)$.

Proof. The proof is similar to Proposition 2 in [11].

This is the first stage of the approximation. As the second stage, from the Theorem (A.5) of [25], we can characterize a measure, say $\mu^{*}$, in the set $Q(M, G)$ at which the function $\mu \rightarrow \mu(g)$ attains its minimum. It follows from a result of Rosenbloom [27], that is:

Proposition 4.2. The measure $\mu^{*}$ in the set $Q(M, G)$ at which the function $\mu \rightarrow \mu(g)$ attains its minimum has the following form

$$
\mu^{*}=\sum_{k=1}^{M+G} \beta_{k}^{*} \delta\left(z_{k}^{*}\right),
$$

with $z_{k}^{*} \in \Omega$ and $\beta_{k}^{*} \geq 0, k=1,2, \cdots, M+G$. Here $\delta_{\Omega}\left(z^{*}\right)$ is unitary atomic measure concentrated at $z^{*} \in \Omega$, characterized by $\delta\left(z^{*}\right)(F)=F\left(z^{*}\right)$, where $F \in C(\Omega)$.

Based on (4.3), the measure theoretical optimization problem (4.1)-(4.2) is equivalent to the following nonlinear optimization problem:

$$
\text { minimize } \quad \sum_{k=1}^{M+G} \beta_{k}^{*} g\left(z_{k}^{*}\right)
$$

subject to

$$
\begin{gathered}
\sum_{k=1}^{M+G} \beta_{k}^{*} k_{i}\left(z_{k}^{*}\right)-u\left(x_{i}\right)=-f\left(x_{i}\right),(i=0,1, \cdots, M), \\
\sum_{k=1}^{M+G} \beta_{k}^{*} \theta_{v}\left(z_{k}^{*}\right)=\alpha_{\theta_{v}}, \quad,(v=1, \cdots, G), \\
\sum_{k=1}^{M+G} \beta_{k}^{*} \leq T, \\
u\left(x_{i}\right) \text { is free, }(i=0,1, \ldots, M), \\
\beta_{k}^{*} \geq 0, \quad(k=1,2, \ldots, M+G),
\end{gathered}
$$

where the unknowns are the coefficients $\beta_{k}^{*}$, supports $z_{k}^{*},(k=1,2, \ldots, M+G)$, and $u\left(x_{i}\right)(i=0,1, \ldots, M)$. It would be computationally convenient if we could minimize 
the function $\mu \rightarrow \mu(g)$ only with respect to the coefficients $\beta_{k}^{*},(k=1,2, \ldots, M+G)$, and $u\left(x_{i}\right)(i=0,1, \ldots, M)$, which leads to a finite-dimensional LP problem. However, we do not know the supports of the optimal measure. The answer lies in a meaningful approximation of this support, by introducing a dense subset in $\Omega$.

Proposition 4.3. Let $\sigma$ be a countable dense subset of $\Omega$. Given $\epsilon>0$, a measure $\bar{\mu} \in M^{+}(\Omega)$ can be found such that

$$
\begin{aligned}
& \left|\left(\mu^{*}-\bar{\mu}\right)(g)\right| \leq \epsilon, \\
& \left|\left(\mu^{*}-\bar{\mu}\right)\left(k_{i}\right)\right| \leq \epsilon, \quad(i=0,1, \ldots, M), \\
& \left|\left(\mu^{*}-\bar{\mu}\right)\left(\theta_{v}\right)\right| \leq \epsilon, \quad(v=1, \cdots, G),
\end{aligned}
$$

the measure $\bar{\mu}$ has the form

$$
\bar{\mu}=\sum_{k=1}^{M+G} \beta_{k}^{*} \delta\left(z_{k}\right),
$$

where the coefficients of $\beta_{k}^{*}$ are the same as in the optimal measure (4.3) and $z_{k} \in \sigma$. Proof. See the proof of Proposition III.3 in [25].

Finally, the above results enable us to approximate the problem via finite dimensional LP problem:

$$
\text { minimize } \quad \sum_{k=1}^{L} \beta_{k} g\left(z_{k}\right)
$$

subject to

$$
\begin{aligned}
& \sum_{k=1}^{L} \beta_{k} k_{i}\left(z_{k}\right)-u\left(x_{i}\right)=-f\left(x_{i}\right),(i=0,1, \cdots, M), \\
& \sum_{k=1}^{L} \beta_{k} \theta_{v}\left(z_{k}\right)=\alpha_{\theta_{v}},(v=1,2, \cdots, G), \\
& \sum_{k=1}^{L+1} \beta_{k}=T, \\
& u\left(x_{i}\right) \text { is free } \\
& \beta_{k} \geq 0,(k=1,2, \ldots, L),
\end{aligned}
$$

where $L>>M+G$ and $z_{k}, k=1, \ldots L$ are fixed in $\sigma$. It is to be noted that we added a slack variable $\beta_{L+1}$ for obtaining equality in (4.7).

In the problem (4.11)-(4.16), $\Omega$ is partitioned into $L$ subregions $\Omega_{1}, \Omega_{2}, \ldots, \Omega_{L}$ where $\Omega=\bigcup_{k=1}^{L} \Omega_{k}$ and $z_{k}$ is chosen in $\Omega_{k}$. To this means, assume that $I=[a, b]$ is divided to 
$m$ portion and $U$ to $p$ portion, that is $L=m p$. In application, the functions $\theta_{v}$ in (4.13) are chosen as piecewise constant. Let us define

$$
\theta_{v}(t, u)= \begin{cases}1 & \text { if } t \in J_{v} \\ 0 & \text { otherwise }\end{cases}
$$

where $J_{v}=\left[\frac{(v-1) T}{m}, \frac{v T}{m}\right], \quad(v=1,2, \ldots, m)$, and we set $G=m$. In the right-hand side of (4.13), $\alpha_{\theta_{v}}$ is the integral of $\theta_{v}(t, u)$ on $[a, b]$; so by (4.17) we have

$$
\alpha_{\theta_{v}}=\int_{J_{v}} \theta_{v}(t, u) d t=\frac{T}{m},(v=1,2, \ldots, m) .
$$

From the above relations and expanding (4.13), we have

$$
\begin{aligned}
\sum_{k=1}^{p} \beta_{k} & =\frac{T}{m}, \\
\sum_{k=p+1}^{2 p} \beta_{k} & =\frac{T}{m}, \\
& \cdot \\
\sum_{k=(m-2) p+1}^{(m-1) p} \beta_{k} & =\frac{T}{m}, \\
\sum_{k=(m-1) p+1}^{m p} \beta_{k} & =\frac{T}{m} .
\end{aligned}
$$

Adding the above equalities leads to

$$
\sum_{k=1}^{L} \beta_{k}=T .
$$

Comparing (4.14) and (4.18) guarantees that $\beta_{L+1}=0$.

From the above analysis, problem (4.11)-(4.16) can be converted to the following LP problem

$$
\text { minimize } \quad \sum_{k=1}^{L} \beta_{k} g\left(z_{k}\right)
$$

subject to

$$
\begin{cases}\sum_{k=1}^{L} \beta_{k} k_{i}\left(z_{k}\right)-u\left(x_{i}\right)=-f\left(x_{i}\right), & (i=0,1, \cdots, M), \\ \sum_{k=1}^{L} \beta_{k}=T, & \\ \beta_{k} \geq 0, & (k=1,2, \ldots, L), \\ u\left(x_{i}\right) \text { is free, } & (i=0,1, \ldots, M) .\end{cases}
$$


An approximating solution for integral equation (1) is construct from the slack variable $u\left(x_{i}\right), i=0,1, \ldots, M$, obtained from the above LP.

\section{$5 \quad$ Numerical examples}

In this section, we propose our method to obtain approximate solution of Fredholm integral equations. Before implementing several test problems, we choose $g(t, u(t))=0$ in the optimization problem (2.2)-(2.3). To compare the solutions we define a error function proposed in [4]:

$$
e\left(x_{i}\right)=u\left(x_{i}\right)-u^{*}\left(x_{i}\right), i=0,1, \ldots, M,
$$

where we suppose $u(x)$ be exact solution of nonlinear Fredholm integral equation (1) and $u^{*}\left(x_{i}\right), i=0,1, \ldots, M$ be a solution obtained by solving the final LP problem.

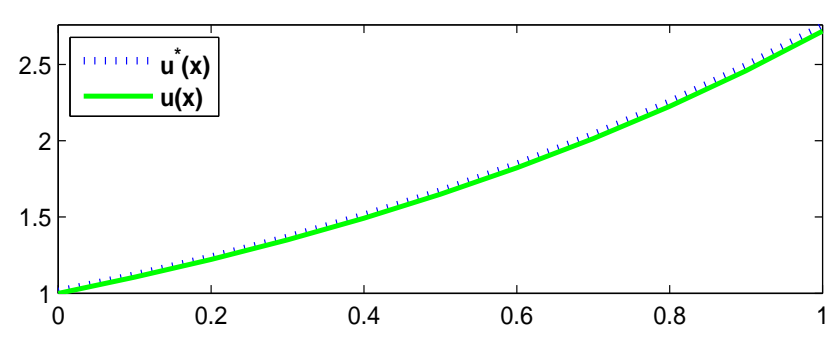

Figure 1: Pointwise curve shows approximate solution and continuous curve shows exact solution.

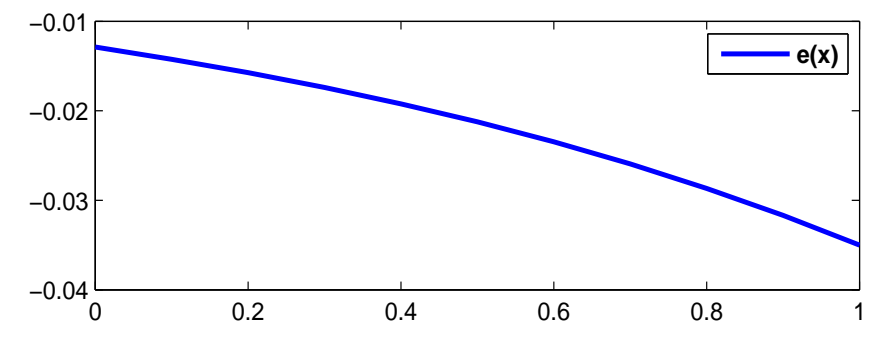

Figure 2: The error function of Example 5.1.

Example 5.1. Consider the following second kind Fredholm integral equation from [2]:

$$
u(x)=e^{x+1}-\int_{0}^{1} e^{x-2 t} u^{3}(t) d t, \quad 0 \leq x \leq 1,
$$




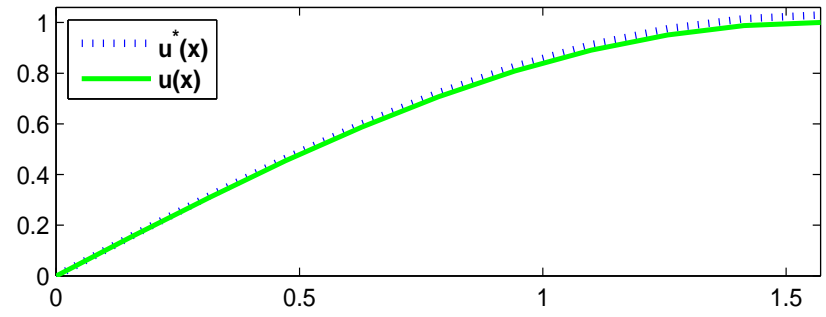

Figure 3: Pointwise curve shows approximate solution and continuous curve shows exact solution.

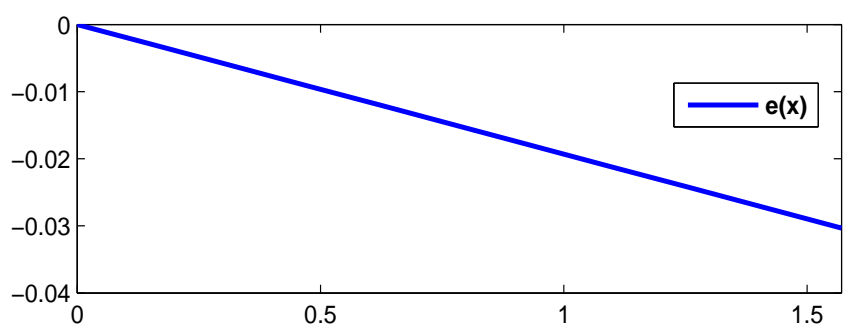

Figure 4: The error function of Example 5.2.

where the exact solution is $u(x)=e^{x}$. We choose $M=10$ and $m=p=50$. Thus $\Omega=$ $[0,1] \times[0,1.57]$ is divided to $N=2500$ equal subintervals. We select $z_{k}=\left(t_{k}, u_{k}\right), k=$ $1,2, \ldots, 2500$, as

$$
k=f+50(e-1), \quad(e, f=1,2, \cdots, 50), \quad z_{k}=\left\{\begin{array}{l}
t_{k}=\frac{1}{50} e \\
u_{k}=\frac{1.57}{50} f .
\end{array}\right.
$$

Thus the corresponding LP model is

$$
\left\{\begin{array}{l}
\text { minimize } \mathbf{0}^{t} \beta \\
\text { subject to } \\
\sum_{k=1}^{2500} \beta_{k} e^{x_{k}-2 t_{k}} u_{k}^{3}+u\left(x_{i}\right)=e^{x_{i}+1}, \quad(i=0,1, \ldots, 10), \\
\sum_{k=1}^{2500} \beta_{k}=1, \\
\beta_{k} \geq 0, \quad k=1,2, \cdots, 2500, \beta^{t}=\left(\beta_{1}, \beta_{2}, \ldots . \beta_{2500}\right) .
\end{array}\right.
$$

One can compare the exact and approximate solutions of the integral equation in Figure 1. The error function (5.1) can be seen in Figure 2. The numerical results are also compared in Table 1.

Example 5.2. As the second example consider the following integral equation considered in [4]:

$$
u(x)=\sin (x)-\frac{x}{4}+\frac{1}{4} \int_{0}^{\frac{\pi}{2}} x t u(t) d t, \quad 0 \leq x \leq \frac{\pi}{2} .
$$


Table 1: The results for Example 5.1 with $x_{i}=\frac{i}{10},(i=0,1 \ldots, 10)$.

\begin{tabular}{cccc}
\hline \hline$x_{i}$ & $u^{*}\left(x_{i}\right)$ & $u\left(x_{i}\right)$ & $e\left(x_{i}\right)$ \\
\hline 0.0 & 1.0129 & 1.0000 & -0.0129 \\
0.1 & 1.1194 & 1.1052 & -0.0142 \\
0.2 & 1.2371 & 1.2214 & -0.0157 \\
0.3 & 1.3672 & 1.3499 & -0.0174 \\
0.4 & 1.5110 & 1.4918 & -0.0192 \\
0.5 & 1.6700 & 1.6487 & -0.0212 \\
0.6 & 1.8456 & 1.8221 & -0.0235 \\
0.7 & 2.0397 & 2.0138 & -0.0259 \\
0.8 & 2.2542 & 2.2255 & -0.0287 \\
0.9 & 2.4913 & 2.4596 & -0.0317 \\
1.0 & 2.7533 & 2.7183 & -0.0350 \\
\hline
\end{tabular}

The analytical solution of this integral equation is $u(x)=\sin (x)$ on $\left[0, \frac{\pi}{2}\right]$. Figure 3 shows that in this example approximate solution tracks the exact one, precisely. The error function in Figure 4 also proves this claim. The numerical results are collected in Table 2 .

Example 5.3. Next example is a Fredholm integral equation of the second kind:

$$
u(x)=\sin \left(\frac{\pi x}{2}\right)-2 x \ln (3)+\int_{0}^{1} \frac{4 x t+\pi x \sin (\pi t)}{u^{2}(t)+t^{2}+1} d t, \quad 0 \leq x \leq 1 .
$$

In this example, $M, m$ and $p$ are also selected the same as Example 5.1. Thus $\Omega=$ $[0,1] \times[0,0.81]$ is divided to $N=2500$ equal subintervals. We select $z_{k}=\left(t_{k}, u_{k}\right), k=$ $1,2, \ldots, 2500$, as

$$
k=f+50(e-1), \quad(e, f=1,2, \cdots, 50), \quad z_{k}=\left\{\begin{array}{l}
t_{k}=\frac{1}{50} e \\
u_{k}=\frac{0.81}{50} f .
\end{array}\right.
$$

The corresponding LP problem is

$$
\left\{\begin{array}{l}
\text { minimize } \mathbf{0}^{t} \beta \\
\text { subject to } \\
-\sum_{k=1}^{2500} \beta_{k} \frac{4 x_{k} t_{k}+\pi x_{k} \sin \left(\pi t_{k}\right)}{u_{k}^{2}+t_{k}^{2}+1}+u\left(x_{i}\right)=\sin \left(\frac{\pi x_{i}}{2}\right)-2 x_{i} \ln (3), \quad(i=0,1, \ldots, 10), \\
\sum_{k=1}^{2500} \beta_{k}=1, \\
\beta_{k} \geq 0, \quad k=1,2, \cdots, 2500, \beta^{t}=\left(\beta_{1}, \beta_{2}, \ldots . \beta_{2500}\right) .
\end{array}\right.
$$

In this example, the analytical solution of the integral equation is $u(x)=\sin \left(\frac{\pi x}{2}\right)$ on $[0,1]$. One may find in Figure 5 the comparison of the obtained exact and approximate 
Table 2: The results for Example 5.2 with $x_{i}=\frac{i \pi}{20},(i=0,1 \ldots, 10)$.

\begin{tabular}{cccc}
\hline \hline$x_{i}$ & $u^{*}\left(x_{i}\right)$ & $u\left(x_{i}\right)$ & $e\left(x_{i}\right)$ \\
\hline 0 & 0.0000 & 0.0000 & 0.0000 \\
$\frac{\pi}{20}$ & 0.1595 & 0.1564 & -0.0030 \\
$\frac{2 \pi}{20}$ & 0.3151 & 0.3090 & -0.0061 \\
$\frac{3 \pi}{20}$ & 0.4631 & 0.4540 & -0.0091 \\
$\frac{4 \pi}{20}$ & 0.5999 & 0.5878 & -0.0121 \\
$\frac{5 \pi}{20}$ & 0.7223 & 0.7071 & -0.0152 \\
$\frac{6 \pi}{20}$ & 0.8272 & 0.8090 & -0.0182 \\
$\frac{7 \pi}{20}$ & 0.9122 & 0.8910 & -0.0212 \\
$\frac{8 \pi}{20}$ & 0.9753 & 0.9511 & -0.0243 \\
$\frac{9 \pi}{20}$ & 1.0150 & 0.9877 & -0.0273 \\
$\frac{\pi}{2}$ & 1.0303 & 1.0000 & -0.0303 \\
\hline
\end{tabular}

solutions. The error function in Figure 6 also shows the precision of the approximate solution. The numerical results are summarized in Table 3.

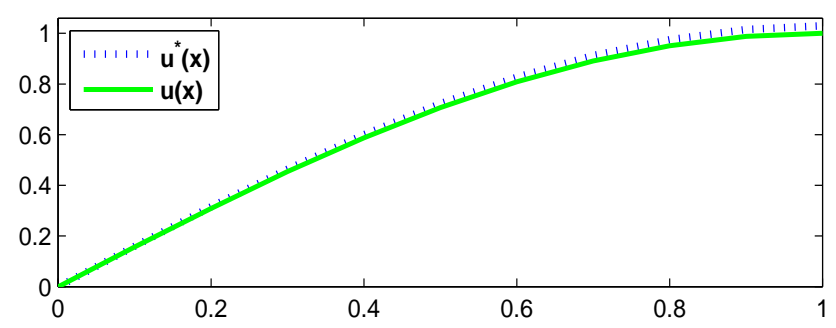

Figure 5: Pointwise curve shows approximate solution and continuous curve shows exact solution.

Example 5.4. The last example is also a Fredholm integral equation of the second kind given in [20] by

$$
u(x)=x^{\frac{1}{2}}-\frac{\pi^{3}}{24} x^{\frac{3}{2}}+\int_{0}^{\frac{\pi}{2}}(t x)^{\frac{3}{2}} u(t) d t, \quad 0 \leq x \leq \frac{\pi}{2} .
$$

The exact solution is $u(x)=x^{\frac{1}{2}}$. We employed again the LP (4.19)-(4.20) and obtained Figures 7 and 8 . The numerical results are briefed in Table 4 .

To end this section, we answer a natural question: are there advantages of our proposed method compared to the existing ones? To answer this, we summarize what 


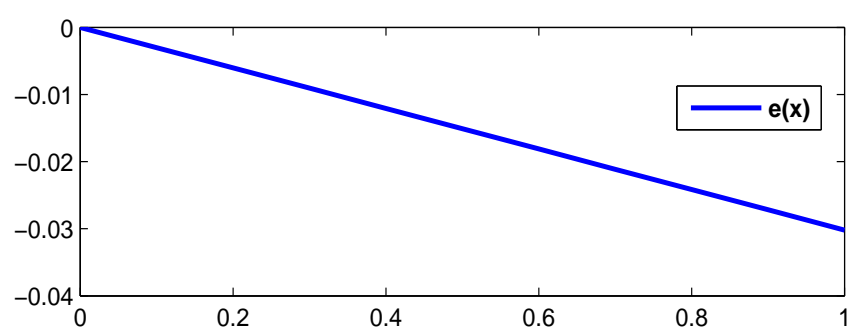

Figure 6: The error function of Example 5.3.

Table 3: The results for Example 5.3 with $x_{i}=\frac{i}{10},(i=0,1 \ldots, 10)$.

\begin{tabular}{lccc}
\hline \hline$x_{i}$ & $u^{*}\left(x_{i}\right)$ & $u\left(x_{i}\right)$ & $e\left(x_{i}\right)$ \\
\hline 0.0 & 0.0000 & 0.0000 & 0.0000 \\
0.1 & 0.1595 & 0.1564 & -0.0030 \\
0.2 & 0.3151 & 0.3090 & -0.0060 \\
0.3 & 0.4631 & 0.4540 & -0.0091 \\
0.4 & 0.5999 & 0.5878 & -0.0121 \\
0.5 & 0.7222 & 0.7071 & -0.0151 \\
0.6 & 0.8271 & 0.8090 & -0.0181 \\
0.7 & 0.9121 & 0.8910 & -0.0211 \\
0.8 & 0.9752 & 0.9511 & -0.0242 \\
0.9 & 1.0149 & 0.9877 & -0.0272 \\
1.0 & 1.0302 & 1.0000 & -0.0302 \\
\hline
\end{tabular}

we have observed from numerical experiments and theoretical results as below:

Comparison of the results of the above examples with those obtained in the corresponding references, shows the efficiency of this algorithm more clearly. This result is intuitive, since the results of this algorithm depend explicitly on the slack variables of the final LP problem (4.19)-(4.20). The proposed transformation method in this article can also allow us to transform easily and efficiently the different kinds of the integral equation problems into an optimization problem. Moreover, since the procedure of this algorithm is not iterative and does not need any initial guess of the solution, subsequently, appears that the applied method in this paper is very easy to use and straightforward in comparison with other numerical methods. 


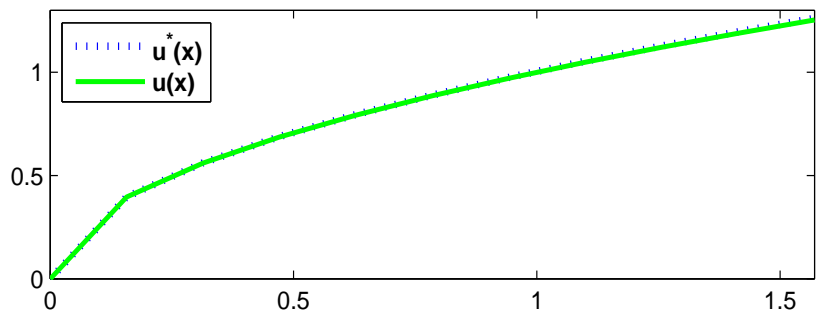

Figure 7: Pointwise curve shows approximate solution and continuous curve shows exact solution.

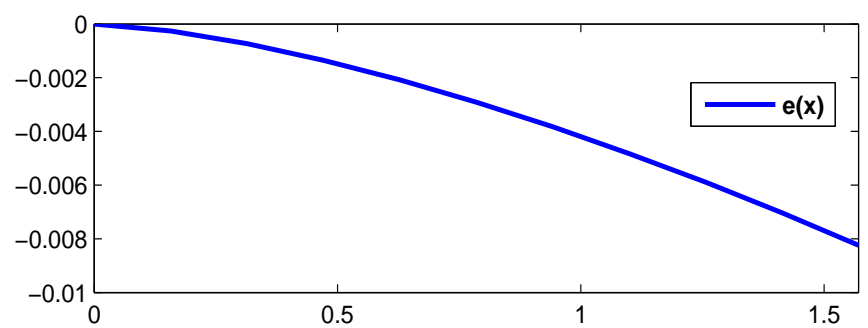

Figure 8: The error function of Example 5.4.

Table 4: The results for Example 5.4 with $x_{i}=\frac{i \pi}{20},(i=0,1 \ldots, 10)$.

\begin{tabular}{lccr}
\hline \hline$x_{i}$ & $u^{*}\left(x_{i}\right)$ & $u\left(x_{i}\right)$ & \multicolumn{1}{c}{$e\left(x_{i}\right)$} \\
\hline 0 & 0 & 0 & 0 \\
$\frac{\pi}{20}$ & 0.3966 & 0.3963 & -0.0003 \\
$\frac{2 \pi}{20}$ & 0.5612 & 0.5605 & -0.0007 \\
$\frac{3 \pi}{20}$ & 0.6878 & 0.6865 & -0.0014 \\
$\frac{4 \pi}{20}$ & 0.7947 & 0.7927 & -0.0021 \\
$\frac{5 \pi}{20}$ & 0.8891 & 0.8862 & -0.0029 \\
$\frac{6 \pi}{20}$ & 0.9746 & 0.9708 & -0.0038 \\
$\frac{7 \pi}{20}$ & 1.0534 & 1.0486 & -0.0048 \\
$\frac{8 \pi}{20}$ & 1.1269 & 1.1210 & -0.0059 \\
$\frac{9 \pi}{20}$ & 1.1960 & 1.1890 & -0.0070 \\
$\frac{\pi}{2}$ & 1.2616 & 1.2533 & -0.0082 \\
\hline
\end{tabular}




\section{Conclusion}

In this paper, we investigated an optimization technique for solving nonlinear Fredholm integral equations of the second kind. The integral equation problem was transformed into an approximating optimization problem, and the embedding method based on some principles of measure theory, functional analysis and linear programming was applied for solving this integral equation. The method is not iterative and it does not need any initial guess of the solution. Furthermore, in this approach the nonlinearity of the continuous kernels has not serious effects on the solution.

\section{References}

[1] K.E. Atkinson. The Numerical Solution of Intrgral Equations of the Second Kind. Cambridge University Press, 1997.

[2] E. Babolian, A. Shahsavaran. Numerical solution of nonlinear Fredholm integral equations of the second kind using Haar wavelets. J. Comput. Appl. Math., 2009, 225: 87 - 95.

[3] C.T. Backer, G.F. Miller. Treatment of Integral Equations by Numerical Methods. Oxford University Press, London, 1977.

[4] A.H. Borzabadi, O.S. Fard. A numerical scheme for a class of nonlinear Fredholm integral equations of the second kind. J. Comput. Appl. Math., 2009, 232: 449 - 454.

[5] C. Cattani, A. Kudreyko. Harmonic wavelet method towards solution of the Fredholm type integral equations of the second kind. J. Appl. Math. Comput., 2010, 215: 4164 - 4171

[6] A. Chakrabarti, S.C. Martha. Approximate solutions of Fredholm integral equations of the second kind. J. Appl. Math. Comput., 2009, 211: 459 - 466.

[7] L.M. Delves, J.L. Mohamed. Computational Methods for Integral Equations. Cambridge University Press, 1985.

[8] L.M. Delves, J. Walsh. Numerical Solution of Integral Equations. Oxford University Press, 1974.

[9] M.H. Farahi, J.E. Rubio, D.A. Wilson. The global control of a nonlinear wave equation. Int. J. Cont., 1996, 65: 1 - 15.

[10] M.H. Farahi, H.H. Mehne, A.H. Borzabadi. Wing drag minimization by using measure theory. J. Optim. Meth. \& Soft., 2006, 21: 169 - 177.

[11] M.H. Farahi, A.H. Borzabadi, H.H. Mehne, A.V. Kamyad. Measure theoretical approach for optimal shape design of a nozzle. J. Appl. Math. \& Comput., 2005, 17: 315 - 328.

[12] C.H. Hsiao. Hybrid function method for solving Fredholm and Volterra integral equations of the second kind. J. Comput. Appl. Math., 2009, 230: 59 - 68.

[13] M. Javidi, A. Golbabai. Modified homotopy perturbation method for solving non-linear Fredholm integral equations. Chaos, Solit. Fract., 2009, 40: 1408 - 1412.

[14] A.J. Jerri. Introduction to Integral Equations with Applications. John Wiley \& Sons, London, 1999.

[15] G. Long, G. Nelakanti. Iteration methods for Fredholm integral equations of the second kind. Comput. \& Math. Appl., 2007, 53: 886 - 894.

[16] G. Long, M.M. Sahani, G. Nelakanti. Polynomially based multi-projection methods for Fredholm integral equations of the second kind. J. Appl. Math. Comput., 2009, 215: 147 155.

[17] K. Maleknejad, T. Lotfi, Y. Rostami. Numerical Compution method in solving Fredholm integral equations of the second kind by using Coifman wavelet. J. Appl. Math. Comput., 
2007, 186: $212-218$.

[18] H.H. Mehne, M.H. Farahi, J.A. Esfahani. On an optimal shape design problem to control a thermoelastic deformation under a prescribed thermal treatment. J. Appl. Math. Modell., 2005, 168: 1258 - 1272 .

[19] R.K. Miller. Nonlinear Volterra Integral Equations. W. A. Benjamin, Menlo Park, CA, 1967.

[20] M. Muhammada, A. Nurmuhammada, M. Moria, M. Sugiharab. Numerical solution of integral equations by means of the Sinc collocation method based on the double exponential transformation. J. Comput. Appl. Math., 2005, 177: 269286.

[21] A.R. Nazemi, M.H. Farahi, H.H. Mehne. Optimal shape design of iron pole section of electromagnet. J. Phys. Lett. A, 2008, 372: 3440 - 3451, doi:10.1016/j.physleta.2008.02.045.

[22] A.R. Nazemi, M.H. Farahi. Control the fibre orientation distribution at the outlet of contraction. J. Acta Appl. Math., 2009, 106: 279 - 292, doi:10.1007/s10440-008-9296-1.

[23] A.R. Nazemi, M.H. Farahi, M. Zamirian. Filtration problem in inhomogeneous dam by using embedding method. J. Appl. Math. \& Comput., 2008, 28: 313 - 332, doi: 10.1007/s12190008-0107-7.

[24] T. Okayama, T. Matsuo, M. Sugihara. Sinc-collocation methods for weakly singular Fredholm integral equations of the second kind. J. Comput. Appl. Math., 2010, 234: 1211 1227.

[25] J. E. Rubio. Control and Optimization; The Linear Treatment of Non-Linear Problems. Manchester, U. K., Manchester University Press, 1986.

[26] W. Rudin. Functional Analysis. Second ed., McGraw-Hill, New York, 1991.

[27] P.C. Rosenbloom. Quelques classes de problems extremaux. Bull. de la Soc. Math. de France, 1952, 80: $183-216$.

[28] A.M. Wazwaz. A First Course in Integral Equations. WSPC, New Jersey, 1997. 